\title{
Editorial: Optical Trapping (Laser Tweezers) and Nanosurgery (Laser Scissors)
}

\author{
Michael W. Berns ${ }^{1,2 \star}$, Halina Rubinsztein-Dunlop ${ }^{3}$, Daryl Preece ${ }^{1,2}$ and Monika Ritsch-Marte ${ }^{4}$ \\ ${ }^{1}$ University of California, Irvine, Irvine, CA, United States, ${ }^{2}$ Beckman Laser Institute and Medical Clinic, Irvine, CA, United States, \\ ${ }^{3}$ School of Mathematics and Physics, The University of Queensland, Brisbane, QLD, Australia, ${ }^{4}$ Department of Physiology and \\ Medical Physics, Medical University of Innsbruck, Innsbruck, Austria
}

Keywords: laser, optics, optical tweezers, trap, laser scissors, cell, tissue

Editorial on the Research Topic

Optical Trapping (Laser Tweezers) and Nanosurgery (Laser Scissors)

This is a diverse compendium of thirty-two research and review articles from an international group of scientists. The common thread that runs through these articles is the use of photons either in the development of photonic technological innovation, or the application of photons through optical trapping (laser tweezers) or laser scissors (nanosurgery) in the alteration/manipulation of living cells. The breadth of contributions should serve as a roadmap for how this optical toolbox can shed light on an array of problems, and in so doing, aid others who may consider using laser tweezers and/or scissors in their research.

\section{TECHNOLOGY DEVELOPMENT}

Nine articles focus on technological aspects of optical trapping or related optical technologies. Several of these deal with accessory approaches to characterize parameters related to how tweezers work. For example, a paper by Dey et al. employs the use of a learning algorithm for tweezer calibration, and Box et al. describe entropy production in an elementary light-driven machine. Other papers examine non-traditional traps that exemplify innovative approaches expanding the traditional traps originally described by Arthur Ashkin. For example, MendozaMuñoz-Pérez et al. describe steady state 3-D trapping using a thermo-capillary, and Pughazhendi et al. describe opto-thermo-electric tweezers and their application. Li et al. describe a hybrid optical array for holographic optical tweezers. In addition, light interaction with non-biological structures are described which eventually may have direct biological and chemical application. For example, Phummirat et al. describe optically controlled gold nanoparticles that may be interfaced with biological systems. This study describes the many uses of optically trapped goldnanoparticles in bioengineering. Optical trapping at this scale has opened up new biological applications.

Received: 05 October 2021

Accepted: 22 October 2021

Published: 16 December 2021

Citation:

Berns MW, Rubinsztein-Dunlop H,

Preece D and Ritsch-Marte M (2021)

Editorial: Optical Trapping (Laser

Tweezers) and Nanosurgery

(Laser Scissors).

Front. Phys. 9:789600.

doi: 10.3389/fphy.2021.789600

\section{BIOLOGICAL APPLICATIONS}

The photonic manipulation of cells and cell-related systems includes a broad spectrum of studies. These studies illustrate the diversity of the investigations by many groups. Some of these studies are hypothesis-driven such as delineating forces inside of cells that affect the process of cell division (mitosis and meiosis) which are outlined in the three papers by Forer et al. which hypothesize and 
test the existence of forces outside of the established role of microtubules to control chromosome movement. Related to this is a review article by Berns covering the use of tweezers and scissors to examine chromosome structure and movements during cell division. Another related perspective review by Blazquez-Castro et al. demonstrates the use of optical trapping and nanosurgery on the manipulation and modification of genetic material. Related to manipulation of genetic material inside of living cells is a brief review paper (perspective) by Kong et al. that reviews the use of laser scissors (nanosurgery) for production of DNA damage followed by delineation of subsequent cellular DNA repair processes. Laser-induced DNA damage and repair studies have been adopted by a significant number of labs around the world and are shedding light on these fundamental cellular processes. The ability of cells to repair damaged DNA and, in particular, defects in the repair process, has direct relevance to such maladies as cancer and birth defects. In addition to the studies on DNA repair using laser scissors, a study by Mo et al. is included, which uses laser tweezers that is applied directly on the DNA molecule in order to study DNA molecule elasticity. In this study, the authors determine trap compliance related to the microspheres that are attached to the DNA. These results will provide valuable calibration information for others who study elasticity of single molecules. Another molecular-based study reported by Riesenberg et al. uses optical trapping to probe receptor-ligand interaction in live cells. These studies and the DNA elasticity studies illustrate how optical traps can be used at the single molecule level as opposed to higher order manipulation of whole cells and organelles.

An area of several papers in this compendium is neuroscience. A paper by Wakida et al. examines changes in $\mathrm{Ca}^{++}$efflux in nervous tissue-derived astrocytes when a nearby astrocyte is killed by laser nanosurgery. Specifically, this study correlates the activation of phagocytosis of dead cell debris by undamaged astrocytes. These results show that astrocytes in nervous tissue play an important role in maintaining tissue homeostasis following damage. In a related study, GomezGodinez examine the role of short-pulsed laser-induced shockwave effects on $\mathrm{Ca}^{++}$in both astrocytes and neurons in vitro. In this study the amount of force generated by the shockwave has been calibrated as a function of the distance of the cells from the shockwave initiation point. In addition to these studies, also using a short-pulsed laser nanosurgery system, Castanares et al. performed nanosurgery on branch specific dendrites. They first analyzed the effect of cutting dendrites in silico and then confirmed in vitro using the ultrafast laser scissors. A dendritic spike evoked in an oblique branch manifested at the soma as an increase in the after- depolarization (ADP). The spikes were branch-specific since not all but only a few oblique dendrites evoked spikes. Experiments showed that cutting certain oblique branches, where dendritic spikes were evoked, curtailed the increase in the ADP. On the other hand, cutting neighboring oblique branches that did not evoke spikes maintained the ADP. The results show that highly targeted dendrotomy can facilitate causal analysis of how branch-specific dendritic spikes influence neuronal output.

Other studies in neurosciences such as the review by Lenton et al. explore the advantages of the use of optical tweezers in providing new and crucial information on neuron dynamics, growth, and communication. One example of such studies is simulation of acceleration and sound at variable frequencies using the combination of OT with Selective Planar Illumination Microscopy (SPIM), to replicate natural vestibular and auditory stimuli. OT offer high spatial precision and therefore enables manipulation of single elements of the inner ear and at the same time precisely map the neural networks that responded, providing important information about the separate and shared circuits involved in hearing and vestibular perception.

In summary, the broad scope of papers in the research topic provide a basis for investigators who are considering the use of optical traps and/or laser nano-ablation scissors in their research. The impact of these optical tools for the study of molecules, cells, and tissue has been considerble, but is still in its infancy with a bright future ahead.

\section{AUTHOR CONTRIBUTIONS}

All authors listed have made a substantial, direct, and intellectual contribution to the work and approved it for publication.

Conflict of Interest: The authors declare that the research was conducted in the absence of any commercial or financial relationships that could be construed as a potential conflict of interest.

Publisher's Note: All claims expressed in this article are solely those of the authors and do not necessarily represent those of their affiliated organizations, or those of the publisher, the editors and the reviewers. Any product that may be evaluated in this article, or claim that may be made by its manufacturer, is not guaranteed or endorsed by the publisher.

Copyright (๑) 2021 Berns, Rubinsztein-Dunlop, Preece and Ritsch-Marte. This is an open-access article distributed under the terms of the Creative Commons Attribution License (CC BY). The use, distribution or reproduction in other forums is permitted, provided the original author(s) and the copyright owner(s) are credited and that the original publication in this journal is cited, in accordance with accepted academic practice. No use, distribution or reproduction is permitted which does not comply with these terms. 